Supplementary Material

\title{
Salt Template Assisted BN Scaffold Fabrication towards Highly Thermally Conductive Epoxy Composites
}

Xuelong Chen ${ }^{\dagger}$, Jacob Song Kiat Lim, Weili Yan ${ }^{\dagger}$, Fang Guo ${ }^{\S}$, Yen Nan Liang ${ }^{\perp}$, Hui Chen ${ }^{\ddagger}$, Alexis Lambourne $^{\#}$, Xiao Hu ${ }^{*}$,, , $\perp$

${ }^{\dagger}$ Rolls-Royce@NTU Corporate Lab, Nanyang Technological University, 50 Nanyang Avenue Singapore 639798; ${ }^{\circledR}$ Temasek Laboratories, Nanyang Technological University, 50 Nanyang Drive, Singapore 637553; ${ }^{\S}$ School of Material Science and Engineering, Nanyang Technological University, Nanyang Avenue, Singapore 639798; ${ }^{\perp}$ Nanyang Environment and Water Research Institute, Nanyang Technological University, Singapore 637141; *Rolls-Royce plc. Central Technology Group, Moor Lane A2 (ML-118), Moor Lane, Derby, UK

*Corresponding email address: ASXHU@ntu.edu.sg 


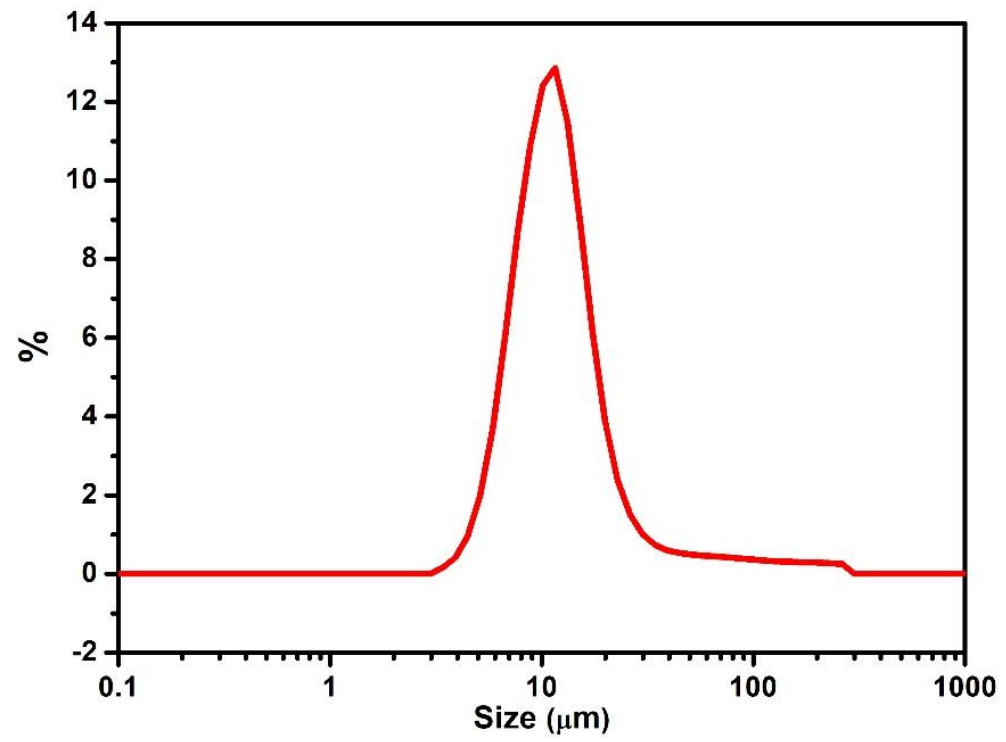

Figure S1. DLS data of BN.

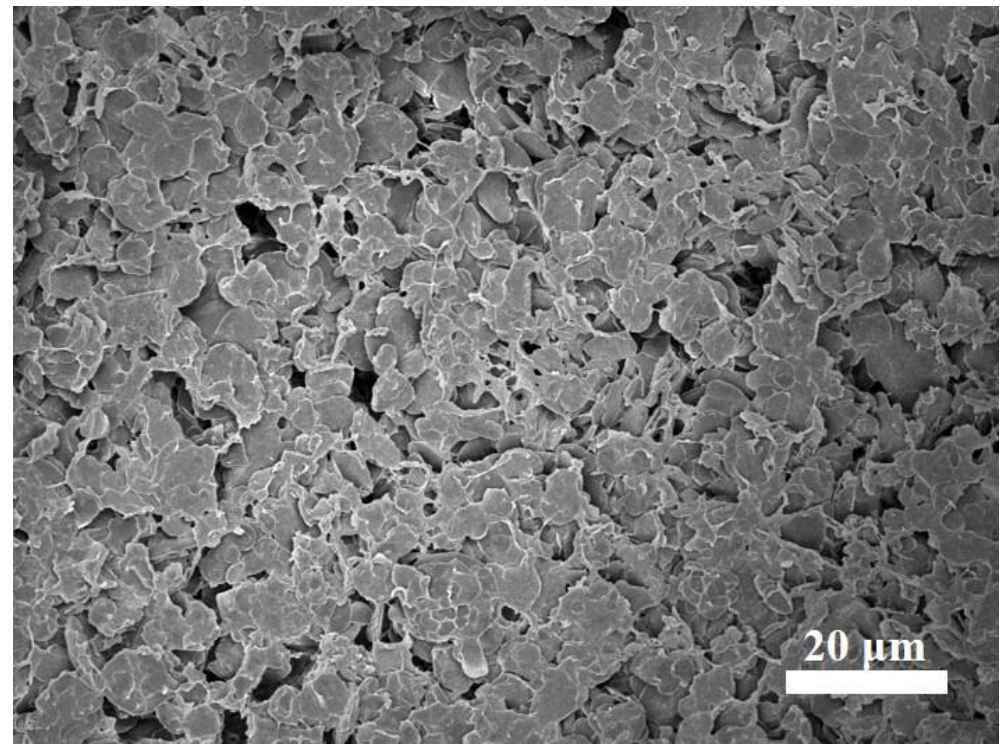

Figure S2. SEM of BN-PVDF scaffold. 


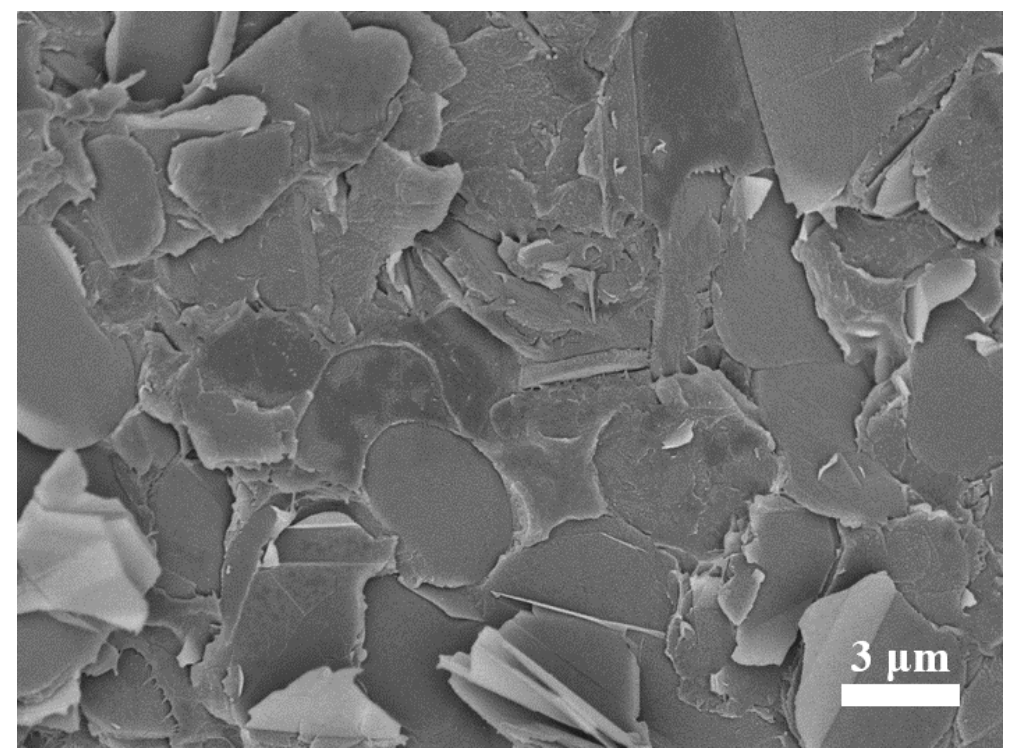

Figure S3. SEM of epoxy/BN-PVDF composite with 16 wt. \% filler loading.

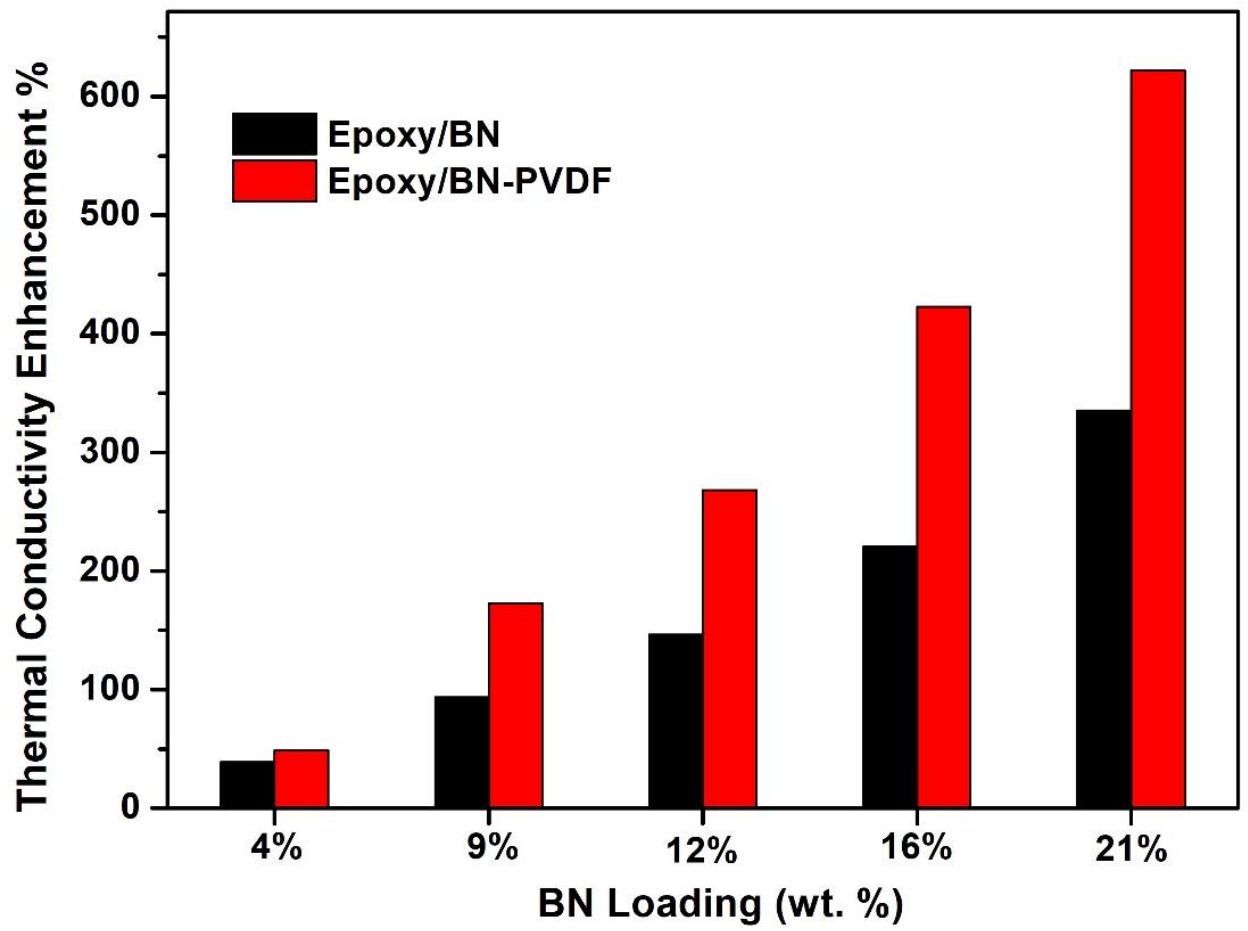

Figure S4. The thermal conductivity enhancement of epoxy composites with and without BN scaffolds. 


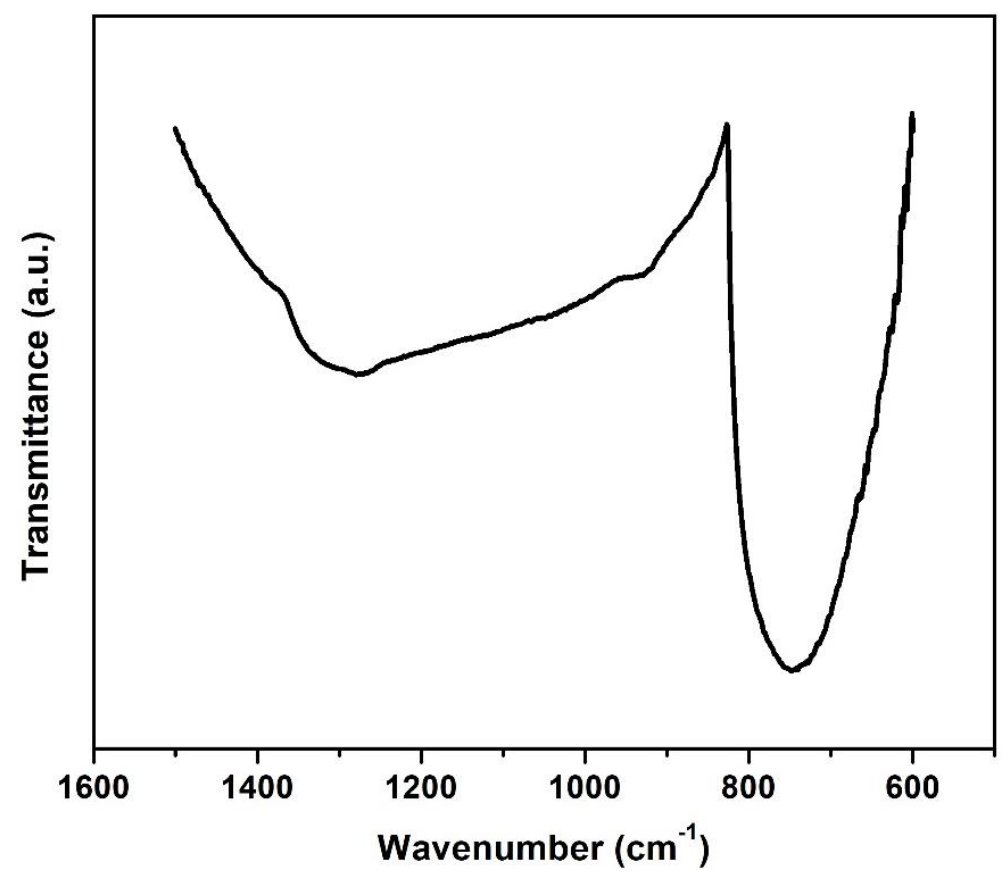

Figure S5. FTIR spectrum of BN. 